\title{
Design of Workshop Forklift Dispatching Management Device
}

\author{
Hongqiang Guo ${ }^{1, a}$, Lijian Zhang ${ }^{1, b}$, Junjie Wang ${ }^{2, c}$ \\ ${ }^{1}$ College of Electrical Engineering, Binzhou Polytechnic,Shandong,256600,China \\ ${ }^{2}$ Bohai Piston Co.,Ltd, Binzhou,Shandong,256600,China \\ axianruibrother@163.com, ${ }^{b} 41350992 @ q q . c o m,{ }^{c} 1653226361 @ q q . c o m$
}

Keywords: Scheduling, Location, Control, Efficiency

\begin{abstract}
In this paper, forklift scheduling management device was designed. The workers can quickly find the right position according to the demand of the forklift, so that the forklift could achieve accurate and efficient scheduling, to prevent sabotage and empty air forklift. And the forklift could work with higher efficiency and stronger control ability; and it can regular parking spaces, improve the management order; With this device, it can make every truck to avoid damage when the forklift cannot find the responsible person.
\end{abstract}

\section{Introduction}

The Zibo textile factory equipped with 8 sets of different performance of the forklift, to carry materials and finished goods transport, unloading, transportation, placement of incoming operations, bear most of the plant short haul work; at the same time, forklift grouping management by different branch room, when the need to be based on the performance of forklift, forklift task the demand for real-time task allocation and scheduling management; and the assessment form on time payment forklift forklift scheduling problem; difficulty, coordination and communication of high cost and low working efficiency, poor ability to control.

The workshop staff often broke into the machine working range, causing damage, and will affect the normal operation of production, personnel location management and alarm can be a very good solution to these problems, ensure the safety of workers.

\section{Device description}

Workshop forklift scheduling management device, including workshop forklift, forklift parking spaces, fork lock, wire pipe and control operation platform (See Fig.1). 


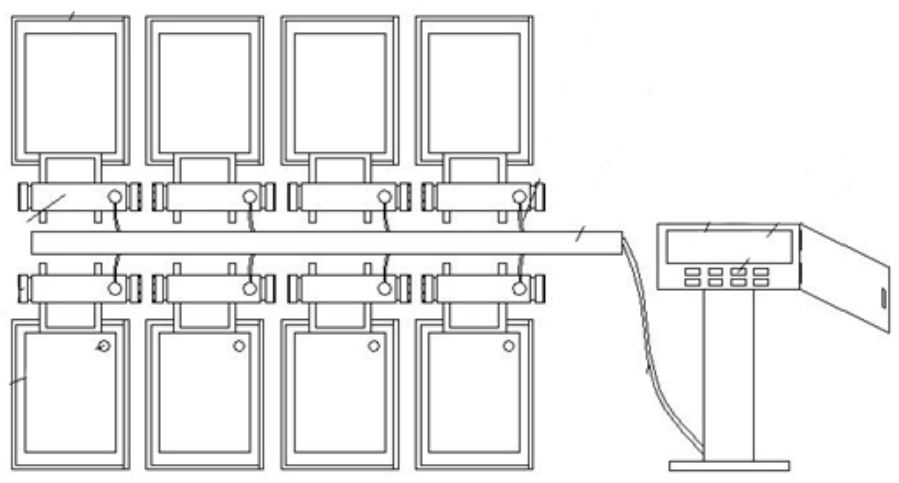

Fig. 1 specific structure distribution

Forklift is provided with a GPS locator, each corresponding to a fork truck parking lock, fork lock includes lock bar, a lock strip on the servo motor drive, cavity and bevel gear transmission device is provided with a locking strip in the output of the servo motor is connected with a driving shaft, a driving shaft is arranged at the other end in the transmission chamber, the bevel gear transmission device comprises a driving bevel gear and a driven bevel gear, a driving shaft connected with the driving bevel gear, a driven bevel gear is connected with the driven shaft, the lock strip is arranged in the compatible with the fork fork fork is provided with a perforation, perforation two, forklift fork is provided with a lock groove fork perforation is arranged on the top of a chute, chute and fork hole is communicated with the slide groove is provided with a lock groove and adapt to phase lock cylinder, the lock is connected with the sliding column, the lock column is arranged on the top of a $\mathrm{N}$ pole magnet piece is arranged on the top of a chute Organic glass, organic glass plate is arranged on the top of a pole pole cavity, cavity is provided with a connecting shaft, the connecting shaft are respectively arranged on both sides of the $\mathrm{N}$ pole and $\mathrm{S}$ pole magnet magnet connected with the driven shaft, the servo motor is provided with a control wire, wire wire inserted in the control tube, the other end of the control wire and control operation the platform is connected, the control operation platform is provided with a network location query terminal and switch card, network location query terminal and GPS locator connected via the mobile communication network, switch card with each fork to lock the corresponding servo motor(See Fig.2).

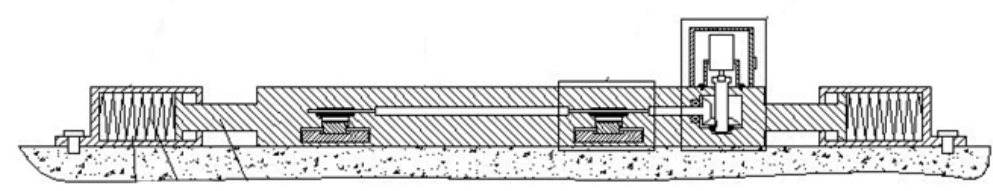

Fig.2Internal structure of fork lock in

A hopper shaped inlet is arranged at the outer end face of the fork hole. 
On both sides of the ground lock strip is provided with a regulating device, adjusting device comprises a casing, and the casing is connected with the ground by the anchor bolt, the shell is provided with a spring cavity, a spring chamber is provided with a telescopic spring lock strip on both sides of the side surface is provided with a telescopic plate, one end of the telescopic plate away from the lock strip extends into the spring cavity, and is connected with a telescopic spring.

The bottom of the driving bevel gear is provided with thrust bearings.

One end of the driven bevel gear is arranged near the driven bevel gear, and the shaft sleeve is far away from the driven bevel gear, and one end of the shaft is provided with a rolling bearing(See Fig.3).

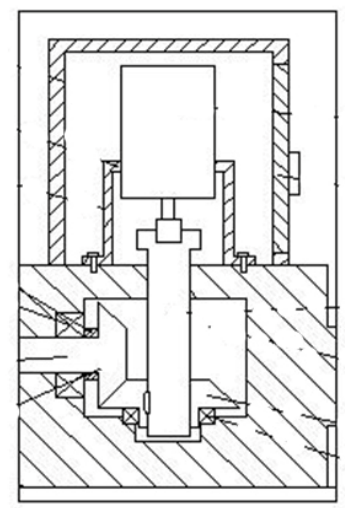

Fig. 3 the inside structure of the fork lock is enlarged

A fixed hoop is arranged on the servo motor, and the fixed hoop hoop is provided with a fixed support fixedly connected with the long strip top surface of the ground lock, and the motor is provided with a motor protecting cover, and the motor protection cover is provided with a box door.

Credit card switch for employee card, credit card switch or ID card.

\section{Device advantage}

1 , the control operation platform is provided with a network location query terminal, forklift is provided with a GPS locator, network location query on the terminal screen can display each truck location in real time, according to the needs of workers can quickly find the right from the display of forklift location, to achieve this position for forklift accurate and efficient scheduling of forklift. The network location query on the terminal screen can display the motion state of the forklift and forklift to prevent empty air, forklift use higher efficiency and stronger control ability. But the device is also provided with a fork lock, forklift driver after work, forklift driver must be forklift fork into the fork lock hole arrival, the fork, which can regular forklift park to the parking, when used again, the first to unlock, then from the forklift fork to remove the lock to use, so it is good to avoid the forklift Loaning loan fang, the forklift can park to the regular parking spaces, improve the management order. Of the forklift when taking place, need to use credit card credit card switch, switch for staff card or ID card switch, so that each truck to the responsibility to the people, to avoid damage when the forklift can not find the responsible person.

2. The outer end face of the fork of the goods fork is provided with a funnel-shaped inlet, which can make the fork of the fork truck easier to insert into the fork of the delivery hole. 
3, when the fork is inserted into the funnel inlet, but not with the fork hole is right, fork and fork perforation have certain deviation, at both sides of the locking strip adjusting device is arranged, in the spring under the action of the telescopic plate can move through the spring cavity expansion. This expansion can move, deviation compensation fork and fork holes exist, the fork easier with fork hole arrival.

4. The thrust bearing at the bottom of the driving bevel gear is used to support the driving bevel gear.

5 , by setting the rolling bearing on the driven shaft, the driven shaft can be rotated more smoothly.

6. The servo motor is provided with a fixed hoop, and the fixing hoop can fix the servo motor better.

7, credit card card switch or switch for employee ID card work card switch, switch to control the servo motor, so that each truck to the responsibility to the people, to avoid damage when the forklift can not find the responsible person.

\section{Working principle}

Control the operation platform of the device 4 is provided with a network location query terminal 24, forklift is equipped with GPS locator 5, signal transmission through the network, the network location query display on the 24 terminal can display each truck location in real time, according to the needs of workers can quickly find the right from the display of the forklift go to the location, location for forklift, to achieve accurate and efficient scheduling of forklift. 24 on the network location query display terminal can display the motion state of the forklift and forklift to prevent empty air, forklift use higher efficiency and stronger control ability.

But the device is also provided with a fork lock 2, forklift driver after work, forklift driver must be forklift fork fork into the arrival of perforation in 13, lock on the fork, so that the forklift can regular can park to the parking, when used again, the first to open the lock, then the forklift from the fork locked out of 2 can be used, so that it is good to avoid the forklift Luantingluanfang, the forklift can park to the regular parking spaces, improve the management order. The device uses a servo motor to control the 7 fork lock switch 2, credit card, 25 control servo motor 7 so the forklift access requires the use of credit card credit card switch 25 and switch 25 for staff card or ID card switch switch, so that each truck can responsibility to the people, to avoid forklift damaged can not find the person responsible.

The device controls the cargo fork ground lock 2 through the servo motor 7, and the credit card switch 25 controls the work of the servo motor 7 . Servo system 7 servo system is mainly located by pulse, servo motor 7 to receive 1 pulses, will rotate the corresponding angle of 1 pulses, so as to achieve angular displacement. The servo motor output end of the drive shaft 7 through 9 will transfer the power to the driving bevel gear 10, a driving bevel gear 10 to transfer the power to the driven bevel gear 11, driven bevel gear 11 to drive the driven shaft 12 through the servo motor 7 receives the pulse Shulaiyi step by step to control the angular displacement of the driven shaft 12 to rotate so, the driven shaft to rotate 180 degrees to 12 each. The connecting shaft 20 is connected with the driven shaft 12, and the driven shaft 12 rotates 180 degrees at a time so as to drive the connecting shaft 20 to rotate 180 degrees.

When the lock on the fork, through the servo motor 7, a bevel gear transmission mechanism step by step to control the connecting shaft 20 , the shaft $20 \mathrm{~N}$ pole magnet 21 lock 
column and 16 at the top of the $\mathrm{N}$ pole magnet piece 17 corresponding, according to the principle of homopolarity repulsion heteropolar phase. The $\mathrm{N}$ pole and $\mathrm{N}$ pole magnet 21 magnets 17 mutually exclusive, so that the lock cylinder 16 moves in the chute within 15, inserted into the lock groove bottom lock fork on the 14, thus completing the locking of the fork.

When the lock fork, through the servo motor 7, a bevel gear transmission mechanism step by step to control the connecting shaft 20 , the shaft 20 S pole magnet 22 lock column and 16 at the top of the $\mathrm{N}$ pole magnet piece 17 corresponding, according to the principle of homopolarity repulsion heteropolar phase. The $\mathrm{S}$ pole and $\mathrm{N}$ pole magnet 22 magnets 17 are attracted to each other, so that the lock cylinder 16 moves in the chute within 15, lock from the lock groove bottom fork on the 14 out, thus completing the fork lock. The plexiglass sheet 18 is provided to separate the runner 15 and the magnetic pole chamber 19.

\section{Conclusion}

Workers can quickly find a suitable forklift according to the demand of the position, to achieve accurate and efficient scheduling of forklift, forklift use higher efficiency, stronger control ability; the forklift can park to the regular parking spaces, improve the management order.

\section{Acknowledgement}

This research was financially supported by Colleges and universities in shandong province department of education scientific research and development projects.

Project name: research and development of intelligent positioning and dispatching management system for forklift truck based on Internet of things. Item number: J17KB133.

\section{Reference}

[1] Dong Jingquan. Intelligent warehouse management system [J]. and MultiAgent networking based on the Sichuan Journal of zoology, 2013 (10): 52-54.

[2] Wang Yanling. Yu Zhaoyu. Analysis of the influence of regional logistics capability on economic growth. Taking Jiangxi Province as an example, Tian logistics technology, [J], 2012,21:277-280.

[3] Zhang Li. Research and application of warehouse management system based on RFID. Logistics engineering and management [J].2014,5:81-83.

[4], Wu Yinghao, Yang Huayun. RFID. Application research in warehouse management. Logistics management, [J].2013,34:26-29 